\title{
La educación en la pandemia: Ampliando las brechas preexistentes
}

Education in the pandemic: Widening the pre-existing gaps

\section{Pablo Chaverri Chaves}

\section{Citar este documento según modelo APA}

Chaverri Chaves, Pablo. (2021). La educación en la pandemia: Ampliando las brechas preexistentes. Revista Actualidades Investigativas en Educación, 21(3), 1-22. Doi. 10.15517/aie.v21i3.46725 


\title{
La educación en la pandemia: Ampliando las brechas preexistentes
}

\author{
Education in the pandemic: Widening the pre-existing gaps
}

\section{Pablo Chaverri Chaves ${ }^{1}$}

\begin{abstract}
Resumen: Este ensayo se ha realizado porque se ha registrado un intenso impacto de la pandemia en la educación $y$, particularmente, en la equidad educativa. Trata acerca de cómo las brechas socioeconómicas perjudican la equidad, la calidad y el rendimiento educativos. Pretende reflexionar sobre la situación del sistema educativo costarricense e internacional antes y durante la pandemia por la enfermedad del covid-19. Se concentra en la forma en que esta crisis influye en las brechas educativas preexistentes de acuerdo con la condición socioeconómica de las personas, para ello se revisan resultados de investigación socioeconómica y sociocognitiva reciente (primordialmente de los últimos 10 años). En primer lugar, se analiza la situación de la desigualdad educativa anterior a la pandemia, luego se identifica un conjunto de factores que permiten comprender las brechas socioeducativas relacionadas con el rendimiento académico y se presenta el caso de los resultados de las pruebas de bachillerato según la condición socioeconómica de estudiantes como un reflejo de tales brechas en el caso costarricense. En segundo lugar, se indaga acerca del impacto de la pandemia en la educación. Finalmente se ofrecen algunas reflexiones concluyentes, basadas en la información recopilada y analizada. Se propone que la actual crisis pandémica ampliará las brechas socioeducativas preexistentes, pues el estudiantado y las familias en pobreza y con carencia de recursos cuentan con condiciones materiales y personales insuficientes para enfrentarla $y$ evitar un creciente rezago educativo.
\end{abstract}

Palabras clave: brechas educativas, desigualdad social, desempeño educativo, pandemia del Covid-19.

Abstract: This essay has been conducted because there has been a registered strong impact of the pandemic on education and, particularly, on educational equity. It is about how socio-economic gaps harm educational equity, quality and performance. It aims to reflect on the situation of the Costa Rican and international education system before and during the Covid-19 pandemic, focusing on how this crisis influences pre-existing educational gaps according to the socioeconomic condition of people, for which results of recent socioeconomic and sociocognitive research (primarily from the last 10 years) are reviewed. First, the issue of educational inequality prior to the pandemic was addressed, then a set of factors that serve to understand the socio-educational gaps related to academic performance were identified and the case of the results of the baccalaureate tests according to the socioeconomic condition of students is presented as a reflection of such gaps in the Costa Rican case. Then it inquired about the impact of the pandemic on education. Finally, some conclusive reflections are offered based on the information collected and analyzed. It is proposed that the current pandemic crisis will widen the pre-existing socio-educational gaps, since students and families in poverty and lack of resources have insufficient material and personal conditions to face it and avoid a growing educational backwardness.

Keywords: educational gaps, social inequality, educational performance, Covid-19 pandemic.

\footnotetext{
1 Docente e Investigador de la Universidad Nacional, en el Instituto de Estudios Interdisciplinarios de la Niñez y la Adolescencia (INEINA), Heredia, Costa Rica. Orcid https://orcid.org/0000-0002-2639-4242
}

Dirección electrónica: pablo.chaverri.chaves@una.cr

Ensayo recibido: 26 de abril, 2021

Enviado a corrección: 10 de junio, 2021

Aprobado: 23 de agosto, 2021 


\section{Introducción}

El sistema educativo costarricense, así como el latinoamericano y el caribeño, ya presentaban importantes brechas y debilidades antes de la pandemia (Busso e Inter-American Development Bank, 2017). Estas se acentuaron con el advenimiento de la crisis sanitaria, provocada por la enfermedad Covid-19, cuyas dimensiones globales han sido evidentes desde marzo de 2020 (Organización para la Cooperación y el Desarrollo Económico [OCDE], 2020). Así pues, este trabajo pretende analizar el problema de la brecha socioeducativa antes y durante la pandemia, para lo cual se basa en dos enfoques fundamentales, el socioeconómico y el neurocognitivo, dentro de los cuales se identifican varios factores claves mencionados a través del texto. El período de tiempo al que se presta mayor atención corresponde al de los últimos diez años, hasta junio de 2021, tiempo en el que se escribe este trabajo.

Como lo demuestra un estudio de González y colegas en el contexto costarricense (González et al., 2020), el rendimiento educativo está asociado con el índice de desarrollo social (IDS) de la comunidad donde las personas residen. En otros términos, obtener buenos resultados académicos no depende solo del esfuerzo individual, sino también de las oportunidades, de los servicios y de los apoyos con los que se dispone en el entorno estudiantil. De acuerdo con este estudio, conforme más alto es el IDS del distrito del que proviene la persona estudiante, mayor es su rendimiento en pruebas académicas estandarizadas (en este caso en las pruebas nacionales de bachillerato de la educación media); de manera que, mientras en el centro del territorio costarricense se concentran las mejores condiciones de vida, también lo tienden a hacer los mejores rendimientos educativos, así como en la periferia del país tienden a bajar los niveles de desarrollo social en conjunto con los resultados de aprendizaje (Artavia-Aguilar et al., 2019).

Estas brechas sociales en el rendimiento educativo se pueden agravar por los efectos de la actual pandemia del Covid-19, a los cuales se suman las consecuencias de la prolongada huelga docente de 2018, cuya duración de 93 días mantuvo inactivo el sistema educativo (Murillo, 2018) en un año lectivo que aspira a 200 días efectivos, aunque ya mostraba problemas de bajo aprovechamiento del tiempo escolar (Programa Estado de la Nación, 2019). Durante este período de huelga, la población estudiantil matriculada en centros educativos públicos prácticamente no recibió lecciones, de ahí que sus procesos de aprendizaje se vieran afectados en gran manera y se comprometieran tempranamente sus posibilidades de continuidad y logro educativo, lo que a su vez pone en riesgo las oportunidades de desarrollo socioeconómico futuro en sus vidas (Berlinski y Schady, 2015). 
Aunque la pandemia continúa en 2021, es previsible considerar que la desigualdad educativa aumentará como producto de esta crisis, que ha impactado de forma negativa a los sectores más vulnerables de la población (OCDE, 2021).

\section{La situación educativa antes de la pandemia}

La situación educativa en Costa Rica, en América Latina y en el Caribe ya era muy desigual desde antes de la pandemia (Busso y Inter-American Development Bank, 2017). Sin embargo, es previsible que esta aumentará la brecha entre quienes poseen acceso a una educación de calidad y quienes no lo tienen (OCDE, 2021). Sin embargo, es importante reconocer que el sistema educativo costarricense es relativamente bueno en su desempeño de aprendizaje esperado comparado con la región de Latinoamérica y el Caribe (OCDE, 2017), lo cual establece una base importante tanto para resistir la pandemia como para impulsar la recuperación.

Dicho lo anterior, uno de los grandes y persistentes retos en el desempeño educativo se relaciona con el nivel académico de su cuerpo docente, pues no puede haber alto logro de aprendizaje sin calidad docente (Busso e Inter-American Development Bank, 2017). No obstante, esta aspiración de alto logro educativo parece todavía lejana, ya que el $74 \%$ de docentes considera la lectura como una práctica obligatoria, poco relacionada con el gusto y el placer, solo un $32 \%$ se siente totalmente preparado para la enseñanza de la lengua. A su vez, la probabilidad de que una persona educadora disponga de los conocimientos y prácticas adecuados para la enseñanza de los procesos de lectura y escritura es del $50 \%$ (Programa Estado de la Nación, 2019).

Las personas docentes que adquirieron más competencias durante su formación universitaria poseen una mayor probabilidad de aplicar prácticas y conocimientos de lectura y escritura establecidos en el programa de estudios (Programa Estado de la Nación, 2019), lo cual constituye una práctica clave para impulsar todo el proceso educativo formal, ya que este se asienta en gran medida en la competencia de lectoescritura (Dehaene, 2018), lo cual incide en el desarrollo de las sociedades, ya que se estima que el aumento en los niveles de competencia lectora en estudiantes es un factor robusto de predicción del crecimiento económico (Kim, Lee y Zuilkowski, 2020). Esto es consistente con la idea de que las sociedades, conforme progresan socioeconómicamente, se tornan más sofisticadas tecnológicamente y, por tanto, cada vez más dependientes del conocimiento especializado, lo 
cual a su vez requiere altas capacidades de práctica y comprensión lectoescritoras (Pinker, 2018).

El modelo de la Enseñanza Basada en la Interacción propone una alternativa para medir el desempeño docente en el aula. En concreto, plantea que este puede captarse apropiadamente en tres dimensiones diferentes, pero complementarias: 1) respaldo emocional, 2) organización del aula y 3) apoyo pedagógico. La primera se refiere a la receptividad de las necesidades, los intereses, las motivaciones y los puntos de vista del y de la infante. La segunda se refiere a las expectativas, las rutinas y la gestión de la conducta proactivas y claras. Por su parte, la tercera se refiere a la retroalimentación de calidad y la promoción de reflexiones de orden superior, que es lo opuesto a la actividad basada en la repetición y la memorización de corto plazo. Cuando estas tres áreas se entrelazan de forma adecuada, se considera que se ha logrado una enseñanza receptiva (Hamre, La Paro, Pianta, y LoCasale-Crouch, 2014).

Pese a que las personas estudiantes aprenden más en aulas con una mejor calidad de interacciones sociales entre quienes participan del proceso de enseñanza, pocas experimentan este tipo de interacciones en su escolarización (Busso e Inter-American Development Bank, 2017). Las y los docentes de América Latina tienden a puntuar a un nivel intermedio en las primeras dos áreas (respaldo emocional y organización del aula), empero a un nivel bajo en la tercera (apoyo pedagógico) (Cruz-Aguayo et al., 2015). Esto impide aprovechar los beneficios de la enseñanza receptiva, la cual se logra cuando las tres dimensiones de la Enseñanza Basada en la Interacción se optimizan. La debilidad mostrada mayormente en el apoyo pedagógico es consistente con los estudios que revelan malos hábitos de estudio en los propios docentes y una débil formación inicial para el ejercicio pedagógico profesional (Programa Estado de la Nación, 2019).

Las posibilidades de contar con una educación receptiva son más bajas conforme más bajo es el nivel socioeconómico del estudiantado, puesto que, precisamente, requieren más apoyo y guía de calidad (Busso e Inter-American Development Bank, 2017). Dada esta relación y el círculo que ella implica, es importante profundizar en su comprensión. A continuación, en la siguiente sección se ofrecen algunos argumentos basados en investigación empírica reciente para comprender por qué quienes provienen de un estatus socioeconómico más bajo tienden a lograr un menor rendimiento académico en relación con quienes provienen de estratos altos. 


\subsection{Factores socioeconómicos y sociocognitivos asociados a un menor logro educativo relativo}

La teoría de la reproducción social de Bordieu y Passeron (2018) es una de las más reconocidas para explicar la desigualdad y la inequidad educativas. Según esta teoría, el sistema educativo no solo contribuye a reproducir la desigualdad, sino que además funciona como un mecanismo para mantenerla o ampliarla, a través de una dinámica de selección de los seleccionados. Es decir, este sistema confirma y legitima una red de privilegios que permiten avanzar y desarrollarse en mayor medida a quienes gozan de mejores condiciones socioeconómicas y culturales; en consecuencia, excluye a quienes poseen las peores condiciones, incluso antes de ingresar a este sistema. Esta teoría propone que la razón por la que se configuran estas brechas educativas reside en el acceso de las personas a los capitales sociales, económicos y culturales, en concreto, a los recursos y a los apoyos que sustentan diferencialmente a unos y otros en su intento por progresar académicamente.

El capital social se refiere al conjunto de personas que funcionan como una red de apoyo para la persona estudiante. Por su parte, el capital económico remite a los recursos materiales a los que se tiene acceso (dinero, bienes, propiedades). A su vez, el capital cultural alude al acceso a recursos lingüísticos y simbólicos que facilitan el ingreso, la permanencia y el éxito educativos. Según Bourdieu y Passeron (2018), el estudiantado posee, de forma desigual, estos capitales, según sus condiciones de origen. Por ende, tal posesión o desposeimiento se encuentra en la base de las posibilidades de logro académico en un sistema que, para este autor, estaría diseñado a la medida de los intereses de los sectores con mayores recursos y, por tanto, con mayor dominio social.

Sin embargo, la igualdad de condiciones en la oferta educativa sin una modificación en las condiciones socioeconómicas previas de las personas puede generar un aprovechamiento muy desigual de la oferta académica, el cual terminaría reproduciendo de forma no intencionada las brechas preexistentes (De la Cruz, 2017). En otros términos, si se igualan las condiciones educativas, pero no se mejoran las condiciones socioeconómicas y familiares de origen de las personas, entonces se puede provocar más bien un ensanchamiento de las brechas, pues quienes provengan de mejores condiciones socioeconómicas estarán también en mejores posibilidades materiales, sociales y culturales de aprovechar oportunidades educativas mejoradas. Por lo tanto, quienes provengan de situaciones más desaventajadas tendrán más dificultades para provecharlas. 
Junto al análisis de los aspectos socioeconómicos de la inequidad educativa, la neurociencia es una de las disciplinas que, en los últimos años, ha estudiado con mayores resultados el origen de la desigualdad académica. De hecho, existe un nuevo campo de investigación bastante desarrollado dentro de esta macrodisciplina, denominado neurociencia de la pobreza. Este ha realizado hallazgos de carácter correlacional acerca de los efectos de la pobreza a nivel cerebral y del sistema nervioso, que pueden ser de utilidad para comprender y eventualmente ayudar a disminuir las brechas educativas asociadas con el nivel socioeconómico de estudiantes.

La neurociencia cognitiva ha encontrado estrechas relaciones entre el nivel socioeconómico y la habilidad cognitiva (medida por el cociente intelectual) y el logro escolar desde la infancia temprana. Dentro de las disparidades destacadas por esta área de investigación (Noble y Giebler, 2020) se encuentran la función ejecutiva (asociada con la corteza prefrontal) y la memoria declarativa (asociada con el hipocampo), las cuales están vinculadas a las brechas en el logro educativo. Particularmente, la pobreza infantil se asocia con altos niveles de estrés y bajos niveles de estimulación cognitiva, eventuales factores causantes de las diferencias en los resultados académicos (Farah, 2017).

La investigación neurocientífica ha mostrado que el estrés afecta el desarrollo de la corteza prefrontal, la función ejecutiva y el hipocampo (crucial en la memoria y la regulación del estrés). Aunado a ello, ha evidenciado que las prácticas de crianza materna amortiguan los niveles de estrés. También ha encontrado que las prácticas de estimulación parental en la infancia temprana predicen el volumen del hipocampo en la adolescencia, así como la respuesta al estrés. En este sentido, las personas adultas de bajo nivel socioeconómico enfrentan enormes barreras para mejorar sus vidas, por lo que es una ironía trágica que generalmente enfrentan mayores dificultades con capacidades disminuidas como resultado de las privaciones experimentadas en sus primeros años de vida. Esta línea de investigación contribuye a comprender mejor los ciclos de reproducción intergeneracional de la pobreza, para así guiar su modificación basada en conocimientos más precisos (Farah, 2018).

Por su parte, la investigación socioeconómica empírica reciente ha producido hallazgos que favorecen la comprensión de la relación entre el estatus socioeconómico y el desempeño académico. Uno de los logros globales de la investigación empírica sobre desigualdad educativa es haber identificado diversos factores que influyen en la brecha de rendimiento y logro académico entre las personas de diferentes condiciones socioeconómicas. Entonces, ¿cómo se pueden sintetizar los hallazgos de la investigación empírica sobre por qué niñas, 
niños y jóvenes de menor nivel socioeconómico tienden a rendir académicamente más bajo que sus pares de mayores ingresos? Los siguientes factores socioeconómicos y sociocognitivos están asociados a un menor logro educativo en las personas estudiantes de bajo estatus socioeconómico:

1. ingresos insuficientes que implican diversas necesidades familiares insatisfechas, lo que impide concentrarse en el proceso educativo (UNESCO, 2020);

2. condiciones de vivienda deterioradas y hacinamiento, lo que dificulta la disponibilidad de espacio y recursos materiales adecuados para estudiar y realizar diversas tareas escolares (Pierse, Carter, Bierre, Law y Howden-Chapman, 2016);

3. trabajo infantil, mayores responsabilidades infantiles domésticas y de sostenimiento económico, factores que disminuyen la disponibilidad de recursos físicos, cognitivos y motivacionales para la educación (Chaverri y Arguedas, 2013);

4. menor estimulación lingüística, menor cantidad de palabras recibidas y menos intercambios lingüísticos con adultos, lo cual muestra un efecto negativo en el desarrollo de la lectoescritura inicial, así como en regiones cerebrales asociadas con el lenguaje (Merz, Maskus, Melvin, He, y Noble, 2020; Romeo, et al., 2018);

5. mayor estrés, mayor inflamación corporal, mayor desgaste del sistema inmune y peor salud, lo cual genera interrupciones en la continuidad del proceso educativo (Noble, Houston, Brito, Bartsch, Kan, 2015; Tawakol, Osborne, Wang, Hammed, Tung, 2019; Troller-Renfree, Brito, Desai, Pooja, Leon-Santos, 2020);

6. mayor preocupación por carencias, menores recursos cognitivos disponibles y peor desempeño cognitivo en la resolución de problemas de razonamiento (Mani, Mullainathan, Shafir y Zhao, 2013);

7. menor capacidad de atención, control inhibitorio y postergación de recompensas inmediatas, capacidades asociadas a períodos de esfuerzo sostenido gradualmente crecientes, necesarios en el proceso educativo (Chaverri, Conejo, León y Arrieta, 2020; Raver, Blair, Willoughby, y Family Life Project Key Investigators, 2013);

8. migración, inestabilidad y desarraigo continuos que generan interrupciones en el proceso educativo, el cual requiere de vínculos afectivos e identitarios sólidos (Stark, Micevska y Mycielski, 2009);

9. jornadas laborales más extensas que alejan más a los miembros de la familia entre sí (padres, madres, hijos e hijas), lo cual disminuye el tiempo y energía disponible para 
intercambios dar-recibir adulto-niño, cuyo papel es crucial en el desarrollo lingüístico y socioemocional del infante (Butcher y Whitmore, 2018);

10. menor acceso a áreas verdes abiertas y recreativas, asociado con la reducción del estrés, una menor incidencia de violencia y una mejor salud mental, factores importantes para la focalización, motivación y aprovechamiento educativo (Nesbitt, Meitner, Girling, Sheppard, y Lu, 2019);

11. menor acceso a centros educativos y docentes de calidad, que suelen ser asignados a las comunidades más centrales y de mejor nivel socioeconómico (Goldhaber, Lavery, y Theobald, 2015);

12. menor acceso a servicios públicos básicos de agua potable, saneamiento, electricidad y transporte, y mayor marginación geográfica, que generan obstáculos y preocupaciones añadidas para las comunidades más empobrecidas, lo cual dificulta la continuidad y tranquilidad necesarias en el proceso educativo (Banerjee y Duflo, 2011);

13. bajo nivel educativo de las figuras parentales, que conlleva menor capacidad de apoyo familiar al proceso educativo de sus hijos (Merz et al., 2020; PEN, 2019);

14. comunidades más conflictivas y menor seguridad ciudadana, lo que implica mayores preocupaciones y peligros tanto en el traslado entre el centro educativo y el hogar, así como durante la permanencia en el centro educativo e incluso en los períodos dentro del hogar, debido a una mayor incidencia de violencia intrafamiliar (Child Trends, 2019);

15. estigmatización, aporofobia y amenaza de estereotipo, que inciden en un menor rendimiento educativo, ya que quienes son señalados y derogados por su condición socioeconómica y étnica muestran menor desempeño en pruebas académicas, posiblemente por dedicar recursos cognitivos y emocionales al prejuicio que reciben (Cortina, 2018; Steele y Aronson, 1995).

Este conjunto de factores, aunque no es exhaustivo, permite afirmar que las condiciones de vida en pobreza configuran una problemática compleja, multifactorial y sistémica, asociada a un menor desarrollo de habilidades sociocognitivas y emocionales necesarias para lograr un rendimiento académico óptimo. A partir de lo anterior, se puede explicar cómo la pobreza se convierte en un predictor de menor rendimiento y logro académico. Estos resultados educativos deficitarios inciden directamente en una mayor dificultad posterior para conseguir empleo o bien para que aquellos empleos que se consigan resulten ser de menor calificación, que están asociados a menores ingresos y peores condiciones laborales, todo lo cual incide 
en la creación de un ciclo de producción y reproducción de la precariedad laboral y la pobreza. Este proceso se presenta esquemáticamente en la figura 1.

Figura 1: ciclo de reproducción de la pobreza

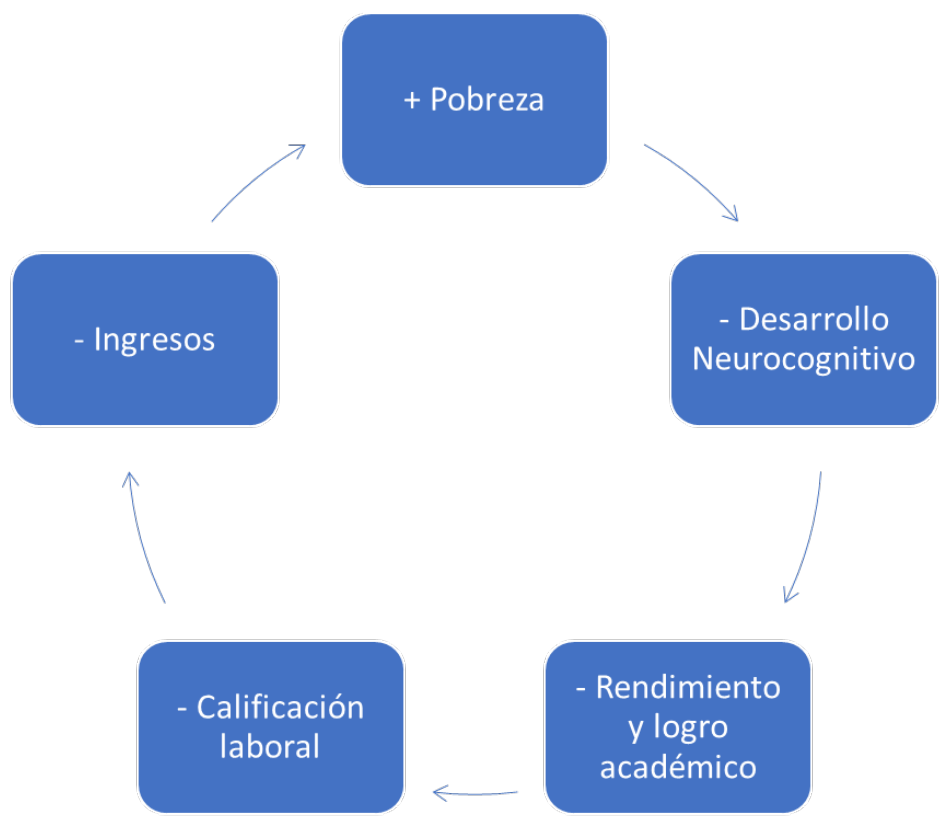

Fuente: elaboración propia con base en la literatura consultada.

Tal como lo describe la figura 1, los impactos negativos de la pobreza inciden negativamente en las posibilidades de rendimiento y logro educativos de las personas estudiantes, lo cual influirá negativamente en sus posibilidades de generación de ingresos. Este círculo de reproducción de la pobreza puede agravarse a causa de los efectos de la pandemia, como se discutirá posteriormente.

La educación es un proceso acumulativo, en el que cada paso depende críticamente de los anteriores, los cuales, a su vez, dependen de contar con un contexto propicio para obtener un logro educativo favorable. En una reciente investigación, González, Acosta, Artavia, Calderón, Chaverri, Cruz, Ramírez, Rodríguez, y Solano (2020) analizan la relación entre el desempeño en las pruebas de bachillerato en la educación media y el distrito de residencia de las personas estudiantes en Costa Rica.

La pobreza, en tanto carencia de recursos materiales para asegurarse un estándar de vida mínimamente aceptable que cubra necesidades básicas, lleva a las personas a disponer de menores recursos cognitivos personales y familiares para enfrentar la demanda académica. La investigación reciente en ciencia cognitiva acerca de la pobreza demuestra que, bajo 
condiciones de carencia material, las personas tienen más dificultad para razonar. Por ejemplo, la investigadora Anandi Mani y sus colegas (Mani, Mullainathan, Shafir y Zhao, 2013) encontraron que el considerar una decisión financiera proyectada, tal como el pagar una reparación de automóvil, afecta el rendimiento de las personas en tareas de razonamiento no relacionadas con este pago. Las personas de bajos ingresos presentaron un desempeño deficiente si las reparaciones eran costosas, pero lo hicieron bien si el costo era bajo, mientras que las personas de mayores ingresos tuvieron un buen desempeño en ambas condiciones, como si la carga financiera proyectada no impusiera presión cognitiva sobre quienes no están en pobreza. Del mismo modo, una muestra de agricultores realizó tareas de razonamiento mejor después de la cosecha que antes; es decir, cuando ya podían contar con suficientes ingresos. Esta investigación sugiere que contar con menores ingresos y recursos perjudica el funcionamiento cognitivo de manera importante.

En otra investigación realizada en Costa Rica (Chaverri, Conejo, León y Arrieta, 2020), se encontró que las personas menores de edad en una condición de bajo estatus socioeconómico (medido por nivel de acceso a bienes y servicios básicos) presentan mayores problemas para esperar en una tarea de postergación de la recompensa. Se concluyó también que aquellos infantes a quienes se les incumple previamente una promesa muestran un menor desempeño para retardar algo que desean. Parece ser que cuando niñas y niños viven en un entorno más impredecible e inseguro tienen dificultades agregadas para autorregular su comportamiento orientándolo a metas que desean conseguir.

El tiempo perdido en educación es difícil de recuperar. En los primeros años, las personas somos más plásticas y si no aprovechamos esa flexibilidad con experiencias nutricias, después ya es mucho más difícil. Es como la diferencia entre abrir una puerta liviana y una bóveda muy pesada, no es que no se pueda abrir la segunda, es que es mucho más difícil. Esto es lo que pasa cuando los primeros años no brindan una experiencia positiva y enriquecedora, sino negativa, estresante y dolorosa.

Las condiciones de inestabilidad, de pobreza y de inseguridad pueden influir en las funciones ejecutivas infantiles (Raver et al., 2013). Esto afecta la capacidad de aprendizaje y el desarrollo. Una investigación reciente en Costa Rica muestra que, cuando niñas y niños se relacionan con otra persona que muestra ser poco confiable, su capacidad de autocontrol sufre; mientras que cuando se relacionan con alguien confiable, entonces pueden lograr mayor autocontrol, entendido como la capacidad de postergar recompensas (Chaverri et al., 2020). 
Así como se ha comprobado que el aumento en el nivel de aprendizaje de la población repercutirá en el crecimiento y desarrollo económicos, también se sabe que la falta de aprendizaje impedirá aprovechar oportunidades (Kim et al, 2020). En este sentido, es importante plantear la siguiente pregunta: ¿dónde están fallando más los ambientes de aula en América Latina?

En una investigación reciente en el contexto latinoamericano, se ha encontrado que las personas docentes puntúan de forma intermedia en aspectos como el apoyo emocional y la organización del aula, pero muy deficientemente en apoyo pedagógico, que depende en gran medida de los conocimientos y las propias prácticas de estudio de las personas docentes (Cruz-Aguayo et al., 2015). Esto es consistente con que tres de cada cuatro docentes no ven la lectura como algo placentero y solo uno de cada tres se considera enteramente preparado para enseñar la lengua (PEN, 2019).

Aunado a la observación anterior acerca de los procesos de aula, a continuación se sintetizan los hallazgos de una investigación reciente en Costa Rica con respecto a la relación entre las condiciones socioeconómicas y los resultados educativos, específicamente en las pruebas de bachillerato al final de la educación media (González et al., 2020), en tanto estos muestran la forma en que el rendimiento académico está ligado a la situación social de las personas, de modo que un empeoramiento en esta última puede empeorar los resultados educativos, por lo cual la pandemia del Covid-19 se coloca como un potencial agravante de la brecha socioeducativa preexistente.

\subsection{Las pruebas nacionales de bachillerato de la educación media y el contexto socioeconómico}

Como se señaló antes, las personas estudiantes tienden a variar en sus resultados académicos en relación con su condición socioeconómica. Una instancia de esta relación se puede ver cuando se analizan los resultados de las pruebas de bachillerato e la educación media en relación con la zona geográfica de residencia de las personas estudiantes. Al respecto, una investigación reciente (González et al., 2020) encontró que el perfil de la población estudiantil que obtiene un mayor rendimiento (más aprobación y mejores notas en los exámenes) en las pruebas nacionales de bachillerato (PNB) en Costa Rica, se caracteriza por lo siguiente: viven en mayor proporción en la Gran Área Metropolitana (GAM) y zona urbana, tienen una edad menor a 18 años, residen en distritos con índice de desarrollo social (IDS) e índice de bienestar de la niñez y la adolescencia (IBINA) medios y altos, su mejor 
calificación la obtienen en la asignatura de inglés y en matemáticas obtienen una diferencia de 13 puntos porcentuales a favor con respecto a los que residen en distritos con IDS de nivel muy bajo. Finalmente, reciben un menor beneficio con la aplicación de la curva, dado que el margen de mejora es menor en comparación con quienes rinden más bajo.

El perfil de la población estudiantil que obtiene menor rendimiento (menor aprobación y menores notas en los exámenes) en las PNB se caracteriza por lo que se describe a continuación: viven fuera de la GAM y zona rural, tienen una edad mayor a los 18 años, residen en distritos con IDS e IBINA de nivel bajo y muy bajo, son más mujeres (solo para 2016), su mejor calificación la alcanzan en la asignatura de biología y la más baja en matemática, y obtienen un mayor beneficio con la aplicación de la curva dado el mayor margen de mejora que tienen con respecto al umbral de aprobación (González et al., 2020).

Considerando el tipo de financiamiento de los colegios, se encontró que los colegios privados y subvencionados muestran los mayores rendimientos y los públicos (con excepción de los científicos, humanísticos y bilingües) los más bajos.

Tomando como referencia el comportamiento de la calificación final de las PNB (suma de la nota de presentación basada en las calificaciones previas -nota de presentación- más la nota obtenida en el examen de bachillerato de cada asignatura) Gonzáles y colegas (2020) lograron, por medio de un análisis de conglomerados, obtener una alta asociación entre el rendimiento de la población estudiantil y las diferentes ofertas educativas, implicando la identificación de tres estratos de colegios de secundaria. Estrato 1: estudiantes de colegios privados, subvencionados, públicos, científicos y experimentales bilingües. Estrato 2: estudiantes de colegios públicos, principalmente académicos diurnos y técnicos profesionales. Estrato 3: estudiantes de colegios rurales, indígenas y colegios no tradicionales. Esta clasificación representa una alternativa viable para analizar las diferencias en el rendimiento del bachillerato según el tipo de centro educativo. Futuras investigaciones podrían ahondar en este aspecto.

Partiendo de la estratificación descrita, se estableció un perfil de las características predominantes en la población estudiantil para cada nivel estructural, las cuales se presentan a continuación. Estrato 1: mayoritariamente mujeres, con promedio de edad de 18,63 años, provienen de distritos cuyo IDS es alto, residen en zona urbana, viven principalmente en la provincia de San José y en la GAM. Estrato 2: mayoritariamente mujeres, con promedio de edad de este estrato de 18,74 años, proviene de distritos cuyo índice de desarrollo social es medio y residen en zona urbana, viven principalmente en las provincias de San José y 
Puntarenas, y en la GAM. Estrato 3: son mayoritariamente mujeres, con promedio de edad de 23,17 años, provienen de distritos cuyo IDS es bajo o muy bajo y residen en zona urbana o rural en aproximadamente la misma proporción, viven principalmente en provincias diferentes a San José y fuera de la GAM (González et al., 2020)

Los anteriores resultados reflejan que las oportunidades y resultados educativos de las personas están fuertemente influidos por el conjunto de condiciones de desarrollo social del contexto en el que viven las personas estudiantes, por lo que al llegar la pandemia a nivel global en 2020, es importante reconocer que se encuentra con personas, comunidades y centros educativos en condiciones muy desiguales entre sí, condicionando esto las posibilidades de resistir, recuperarse y superar la crisis sanitaria del Covid-19. Los factores socioeconómicos aquí identificados, que están a la base de las brechas educativas, reflejan una serie de fragilidades relativas que hacen que la pandemia perjudique más a quienes ya estaban peor, pues "cuando el agua sube, no todos flotan igual", ya que, siguiendo esta metáfora, algunos están en barco, otros en lancha, otros en flotador y otros sin recurso alguno.

Luego de revisarse la problemática de las brechas socioeconómicas en la educación antes de la pandemia, se pasa ahora a la revisión de la forma en que la pandemia estaría ahondando tales brechas educativas.

\section{El impacto de la pandemia en la educación}

Según reportó la UNESCO al inicio de la pandemia (2020), más de 1.500 millones de estudiantes en 188 de 206 países no han podido salir a sus centros educativos debido al COVID-19, lo que representa más del $91 \%$ de la población estudiantil mundial. La pandemia ha puesto de manifiesto y ampliado las enormes disparidades en el acceso a Internet para niños y jóvenes, así como la disponibilidad de recursos, materiales y oportunidades de aprendizaje.

En criterio de la Organización de las Naciones Unidas (2020), los cierres de centros educativos pueden afectar desproporcionadamente a niños y jóvenes que ya experimentan barreras para acceder a la educación, o que corren un mayor riesgo de ser excluidos por una variedad de razones, entre ellas figuran las discapacidades, la residencia en lugares remotos con menor o nulo acceso a internet, el ser migrante, así como pertenecer a familias que han perdido ingresos como resultado de la merma parcial o completa de su empleo.

Las personas estudiantes que viven en los lugares más desconectados del mundo también se enfrentan a la internet menos estable, más lenta y a los precios menos asequibles. 
Para la ONU (2020), aunque la brecha digital se ha ido cerrando en el mundo, más del 46\% de las personas siguen sin acceso, y entre las mujeres, la tasa es menor, con más de la mitad de todas ellas fuera de línea. La falta de acceso a la tecnología e internet se basa en las desigualdades preexistentes entre los diversos grupos y estratos sociales en materia de ingresos, recursos materiales, acceso a la atención sanitaria, electricidad y agua potable, condiciones de vida y de trabajo, acceso a la protección social y educación de calidad.

La ONU (2020) estima que 3.600 millones de los 7.800 millones habitantes del mundo permanecen desconectadas de internet hoy en día, y la mayoría de ellas en los países subdesarrollados. Sólo el $30 \%$ de los países de bajos ingresos pueden proporcionar acceso a la formación digital a sus estudiantes. Antes de esta crisis, casi un tercio de los niños y jóvenes del mundo ya estaban excluidos digitalmente.

Por su parte, UNICEF (2020) plantea que las discapacidades y las necesidades especiales son especialmente difíciles de atender a través de programas a distancia. Las pérdidas serán mayores para los estudiantes que, debido a la pandemia, se ven presionados a abandonar por completo la educación formal. Esta posibilidad se hace mayor entre más tiempo se cierran las escuelas y más profunda es la contracción socioeconómica causada por la pandemia.

Dado que el distanciamiento físico es una medida clave para contener el virus, y el acceso en línea es la principal manera de mantenerse conectado, las y los estudiantes con menor acceso a internet y tecnología apropiada se ven mayormente marginados de las oportunidades educativas.

\subsection{Conectividad y educación en Costa Rica}

La pandemia ha convertido la conectividad a internet en un aspecto crítico para la continuidad de los procesos educativos que se realizan a distancia, pero el acceso territorial diferenciado a internet, la poca preparación tecnológica de muchas familias, la baja capacitación de muchos docentes y la falta de dispositivos y espacios adecuados y suficientes, generan una situación de ampliación de las brechas educativas preexistentes.

De acuerdo con la Encuesta Nacional de Hogares del 2019, un 67\% de estudiantes en la Región Central tenía conexión a Internet desde sus hogares. Un $29 \%$ solo tenía acceso a través del celular y un $3 \%$ no tenía ninguna conexión. Mientras tanto, en las regiones periféricas la conexión desde el hogar era del $40 \%$ y cerca de un $10 \%$ no tenía ninguna conexión a internet (Instituto Nacional de Estadística y Censos [INEC], 2019). 
La falta de espacio suficiente en los hogares para el estudio a distancia y el teletrabajo produce hacinamiento físico, que aumenta conforme más estudiantes tenga la casa. Al respecto, un $4,8 \%$ de los hogares con dos estudiantes tiene problemas de hacinamiento por dormitorio, cifra que aumenta a un $10 \%$ si hay tres estudiantes y a $36 \%$ cuando hay cuatro o más estudiantes (Mata, Trejos y Oviedo, 2021).

Por otro lado, se considera hacinamiento tecnológico cuando la relación es de tres o más personas por cada aparato tecnológico en el hogar (incluyendo computadora de escritorio o portátil, tableta o celular). Los hogares con mayores ingresos tienen en promedio una mayor disponibilidad de tabletas y computadoras portátiles. $Y$ en la región central del país se encuentran los mayores promedios de tenencia de dispositivos por hogar (Mata et al., 2021).

El hacinamiento tecnológico perjudica al 3\% de los hogares costarricenses, pero sube al $14 \%$ en los hogares con cuatro estudiantes o más. Excluyendo el celular, el hacinamiento tecnológico llega al $51 \%$ de los hogares del país, mientras es del $67 \%$ en los hogares con tres estudiantes y del 77\% en aquellos con cuatro o más estudiantes (Mata et al., 2019).

Una encuesta en línea a 42.074 docentes de centros educativos públicos en todo el país, aplicada por el Informe Estado de la Educación del Programa Estado de la Nación (PEN), en conjunto con el Ministerio de Educación Pública (MEP) entre el 23 de abril y el 15 de mayo de 2020 , encontró que un $25 \%$ del personal tiene conexión inestable o que no tienen del todo, de los cuales un $2 \%$ de docentes está sin conexión a internet, constituido por 751 profesores y profesoras que se concentran en direcciones regionales fuera del GAM. Las zonas alejadas y más vulnerables tienen mayor dificultad de interacción por problemas de conexión adecuada (PEN, 2020).

Asimismo, según esta misma encuesta, poco más de la mitad del profesorado no cuenta con formación en herramientas virtuales para la enseñanza, ni en la universidad ni a lo largo del ejercicio de su carrera profesional, es decir que no tienen conocimientos para dar clases a distancia.

Las brechas de conectividad suman varios obstáculos que amplían la desigualdad educativa: conexión a internet desigual en el territorio, espacio físico y recursos tecnológicos desiguales, preparación familiar desigual para apoyar a sus estudiantes, y docentes poco preparados para trabajar a distancia.

Las personas de nivel socioeconómico más bajo se ven más perjudicadas por la pandemia del COVID-19 que quienes tienen una situación más ventajosa, debido a que las primeras tienen menos recursos y posibilidades para hacerle frente a la misma, tanto en su 
entorno, como a nivel personal. Esta desventaja relativa puede resultar en una ampliación de las brechas preexistentes a la pandemia.

\section{Conclusión}

La actual crisis pandémica, muy posiblemente, ampliará las brechas socioeducativas preexistentes, pues estudiantes y familias en situación de pobreza y con carencia de recursos cuentan con condiciones materiales y personales insuficientes para enfrentarla y evitar un creciente rezago educativo. Como se ha evidenciado en este ensayo, se puede afirmar que la pandemia impacta de forma diferente a las personas estudiantes, pues perjudica más a quienes, desde antes de la pandemia, contaban con menos recursos familiares, sociales, económicos, culturales y cognitivos para hacerle frente a esta crisis. De ahí que sea previsible una ampliación de las brechas socioeducativas preexistentes. Si el sistema educativo, social y económico no consideran estas brechas, es más probable que terminen amplificándolas, tanto por la falta de respuestas oportunas, como por la generación de respuestas inadecuadas a tales desigualdades.

Estar bien educado hoy no es solo disponer de un conjunto de herramientas técnicas, sino también estar fortalecido en todas las áreas del ser humano, puesto que hacer frente a los grandes retos del mundo actual requiere tanto de conocimientos como de actitudes, emociones, valores, y habilidades diversas, así como de condiciones materiales óptimas. Para contribuir a cerrar las brechas socioeducativas, la educación debe trabajar estas diversas competencias y condiciones, de ahí que la visión tecnocrática-unidimensional de la educación resulta descontextualizada, reduccionista y reproductora de la desigualdad.

Los grandes retos del siglo XXI requieren que el derecho a la educación no se límite solo al acceso, sino que incorpore la calidad para todas las personas, junto a la equidad, puesto que para pasar de un sistema de baja demanda cognitiva (memorístico-repetitivo) como el actual, a otro de alta demanda (crítico-reflexivo-práctico), es necesario trabajar paralelamente en el mejoramiento de las condiciones socioeconómicas, familiares, afectivas, comunicativas y culturales en las que se desenvuelve la niñez desde sus primeros años de vida.

Por consiguiente, es fundamental lograr una comprensión integral de la calidad educativa en el contexto de una visión integral del desarrollo humano. El reduccionismo tecnocrático opera en contra de sus propias pretensiones, pues declara buscar el progreso y el mejoramiento de la vida humana, pero se dirige solamente a los aspectos técnicos, dejando de lado las dimensiones éticas, sociales, emocionales, creativas, humanísticas y ambientales. 
Los grandes cambios que requiere el sistema educativo no son posibles sin un cuerpo docente alta e integralmente calificado. Esto implica tanto la depuración de los sistemas de reclutamiento, de selección, de nombramiento y de carrera docente, al igual que la mejoría sustantiva de la formación universitaria inicial de las nuevas personas pedagogas, quienes requieren de conocimientos y herramientas para la inclusión social, así como de los apoyos y servicios necesarios para que nadie se quede atrás, de modo que se pueda revertir el círculo perverso presentado aquí en la figura 1.

El logro de un sistema educativo equitativo y de calidad en el futuro no ocurrirá por inercia, sino que será el producto de las decisiones que se tomen hoy como sociedad, así como la educación que se tiene hoy es el producto de las decisiones tomadas en el pasado. Costa Rica se preocupó por ampliar la matrícula, pero no procuró mejorar la equidad ni la calidad, lo cual explica, en parte, las amplias brechas existentes en la actualidad, que han impedido a grandes sectores de la población acceder a oportunidades laborales más calificadas, tal como lo refleja la figura 1. En educación los aciertos son muy favorables a largo plazo y los desaciertos cuestan muy caro y es difícil recuperarlos después.

Durante mucho tiempo se entendió la calidad y el rendimiento educativos como opuestos a la equidad y la inclusión. Sin embargo, hoy, gracias a los avances investigativos reseñados aquí, se comprende que son dimensiones complementarias, en la medida en que el desarrollo del mayor potencial humano no solo depende del esfuerzo y la capacidad individuales, sino también del contexto socioeconómico, familiar, cultural, emocional y lingüístico en el que se vive, como lo muestran los factores identificados en este trabajo.

Nadie puede poseer éxito pleno en una sociedad desigual e injusta, así como nadie debería satisfacerse con el hecho de que hoy muchos niños, niñas y jóvenes son privados de desarrollar todo su potencial, en especial por el conjunto de las circunstancias socioeconómicas en las que les tocó nacer y crecer, y no necesariamente por factores individuales.

Aunque el discurso oficial reconoce la importancia crucial de la educación, el sistema educativo costarricense y el latinoamericano presentan todavía una calidad y una equidad deficientes. Es paradójico que, por un lado, se reconozca el valor crucial de la educación de calidad para todos y todas, mientras que, por otro, se considere culturalmente la profesión educativa como una disciplina de "segunda". Para gozar de una educación de alta calidad no bastan la intuición y las buenas intenciones, sino que es necesario el desarrollo de la investigación científica del mayor nivel posible para contextualizarla y conducirla hacia una 
mayor eficacia, una mayor equidad y un mayor impacto en el desarrollo humano. Los avances científicos recientes en investigación socioeconómica y sociocognitiva son herramientas para una comprensión más profunda y detallada de los factores que perjudican o potencian el rendimiento educativo, los que en su conjunto pueden conducir a diagnósticos y acciones más acertadas.

Como se vio en este trabajo, el aprovechamiento de las oportunidades educativas está ligado a las condiciones socioeconómicas en las que viven las personas estudiantes, razón por la cual no será posible aspirar a lograr un sistema educativo de alta calidad y desempeño mientras no se trabaje de forma paralela en el mejoramiento de tales condiciones. Mucha de nuestra capacidad como sociedad de aprovechar las oportunidades y eludir los riesgos que traerá el resto del siglo XXI dependerá de que comprendamos la forma en que las condiciones socioeconómicas, la educación y las posibilidades de desempeño de las personas están inevitablemente ligadas, de modo que podamos avanzar hacia una sociedad más equitativa, basada en tal comprensión.

\section{Referencias}

Artavia-Aguilar, Cindy V., Calderón-Ramírez, Guillermo., Ramírez-González, Andrea., González-Alvarado, Francisco., Chaverri-Chaves, Pablo., y Cruz-Arroyo, Eddy. (2019). Análisis de resultados de las pruebas nacionales de bachillerato de educación media de los colegios que conforman las direcciones regionales de educación en zonas fronterizas costarricenses. 2013-2016. Revista Electrónica Educare, 23(3), 1-23. https://doi.org/10.15359/ree.23-3.1

Banerjee, Abhijit V., y Duflo, Esther. (2011). Poor economics: A radical rethinking of the way to fight global poverty. Public Affairs.

Berlinski, Samuel., y Schady, Norbert (Eds.). (2015). Los primeros años: El bienestar infantil y el papel de las políticas públicas. Inter-American Development Bank.

Bourdieu, Pierre., y Passeron, Jean. (2018). La reproducción: elementos para una teoría del sistema educativo. Siglo Veintiuno.

Busso, Matías., e Inter-American Development Bank. (2017). Aprender mejor: Políticas públicas para el desarrollo de habilidades. Banco Interamericano de Desarrollo.

Butcher, Kristin., y Whitmore, Diane (2018). Most Workers in Low-Wage Labor Market Work Substantial Hours, in Volatile Jobs. Center on Budget and Policy Priorities.

Chaverri, Pablo., y Arguedas, Ana. (2013). Identificación de personas menores de edad que laboran en los distritos de Mercedes, San Francisco y Vara Blanca (Informe de investigación). Heredia, Costa Rica: Instituto de Estudios Interdisciplinarios de la Niñez y la Adolescencia, Universidad Nacional. 
Chaverri, Pablo., Conejo, Luis D., León, Sara., y Arrieta, Luis A. (2020). Postergación de la Gratificación en Preescolares Costarricenses: Efecto de la Confianza en la Persona Experimentadora y del Estrato Socioeconómico [Informe de investigación]. Heredia, Costa Rica: Instituto de Estudios Interdisciplinarios de la Niñez y la Adolescencia, Universidad Nacional.

Child Trends. (2019). Adverse childhood experiences. Recuperado de https://www.childtrends.org/?indicators=adverse-experiences

Cortina Orts, Adela. (2018). Aporofobia, el rechazo al pobre: Un desafío para la democracia. Barcelona: Paidós.

Cruz-Aguayo, Yyannú., LoCasale-Crouch, Jennifer., Schodt, S., Guanziroli, T., Kraft-Sayre, M., Melo, C., Hasbrouck, S., Hamre, B., and Pianta, R. 2015). Early schooling classroom experiences in Latin America: Focusing on what matters for children's learning and Development. BID.

De la Cruz, Gabriela. (2017). Igualdad y equidad en educación: retos para una América Latina en transición. Educación, 26(51), 159-178. Recuperado de http://www.scielo.org.pe/scielo.php?script=sci arttext\&pid=S1019-94032017000200008

Dehaene, Stanislas. (2018). El cerebro lector: Últimas noticias de las neurociencias sobre la lectura, la enseñanza, el aprendizaje y la dislexia. Buenos Aires: Siglo Veintiuno Editores.

Farah, Martha. (2017). The Neuroscience of Socioeconomic Status: Correlates, Causes, and Consequences. Neuron, 96(1). Doi 10.1016/j.neuron.2017.08.034

Farah Martha. (2018). Socioeconomic status and the brain: prospects for neuroscienceinformed policy. Nature Reviews Neuroscience, 19. Doi https://doi.org/10.1038/s41583$\underline{018-0023-2}$

Goldhaber, Dan., Lavery, Lesley., and Theobald, Roddy. (2015). Uneven Playing Field? Assessing the Teacher Quality Gap Between Advantaged and Disadvantaged Students. $\begin{array}{llll}\text { Educational } & \text { Researcher, 293-307. Doi }\end{array}$ https://doi.org/10.3102/0013189X15592622

González-Alvarado, Francisco., Acosta-Ballestero, Ignacio., Artavia-Aguilar, Cindy., CalderónRamírez, Guillermo., Chaverri-Chaves, Pablo., Cruz-Arroyo, Eddy., Ramírez-González, Andrea., Rodríguez-Pineda, Magaly., y Solano-Alpízar, José. (2020). Hallazgos principales de la investigación "Balance crítico de las Pruebas Nacionales de Bachillerato en Educación Media como instrumento de certificación del conocimiento del estudiantado". Revista Electrónica Educare, 24(1), 1-24. https://doi.org/10.15359/ree.24$\underline{1.13}$

Hamre, Bridget., La Paro, Karen., Pianta, Robert y LoCasale-Crouch, Jennifer. (2014). Classroom Assessment Scoring System (CLASS) Manual. Brookes Publishing. 
Instituto Nacional de Estadística y Censos (INEC). (2019). Encuesta Nacional de Hogares del 2019. San José, Costa Rica: INEC.

Kim, Young-Suk G., Lee, Hansol., and Zuilkowski, Stephanie S. (2020). Impact of Literacy Interventions on Reading Skills in Low- and Middle-Income Countries: A Meta-Analysis. Child Development, 91(2), 638-660. https://doi.org/10.1111/cdev.13204

Mani, Anandi, Mullainathan, Sendhil., Shafir, Eldar., \& Zhao, Jiaying. (2013). Poverty Impedes Cognitive Function. Science, 341(6149), 976-980. Doi https://doi.org/10.1126/science.1238041

Mata, Catherine.,Trejos, Juan., y Oviedo, Luis (2021). Vulnerabilidad tecnológica y económica del estudiantado en Costa Rica en tiempos del COVID19. Ponencia preparada para el Octavo Informe del Estado de la Educación, 2021.

Merz, Emily C., Maskus, Elaine A., Melvin, Samantha A., He, Xiaofu., and Noble, Kimberly G. (2020). Socioeconomic Disparities in Language Input Are Associated With Children's Language-Related Brain Structure and Reading Skills. Child Development, 91(3), 846860. https://doi.org/10.1111/cdev.13239

Murillo, Álvaro. (2018). Una huelga interminable dejó abierta la puerta de las aulas. Semanario Universidad. Recuperado de https://semanariouniversidad.com/pais/una-huelgainterminable-dejo-abierta-la-puerta-de-las-aulas/

Nesbitt, Lorien., Meitner, Michael J., Girling, Cynthia., Sheppard, Stephen R. J., and Lu, Yuhao. (2019). Who has access to urban vegetation? A spatial analysis of distributional green equity in 10 US cities. Landscape and Urban Planning, 181, 51-79. Doi https://doi.org/10.1016/j.landurbplan.2018.08.007

Noble, Kimberly G., Houston, Suzanne M., Brito, Natalie H., Bartsch, Hauke., Kan, Eric., Kuperman, Joshua M., Akshoomoff, Natasha., Amaral, David G., Bloss, Cinnamon S., Libiger, Ondrej., Schork, Nicholas J., Murray, Sarah S., Casey, B. J., Chang, Linda., Ernst, Thomas M., Frazier, Jean A., Gruen, Jeffrey R., Kennedy, David N., Van Zijl, Peter., Sowell, Elizabeth R. (2015). Family income, parental education and brain structure in children and adolescents. Nature Neuroscience, 18(5), 773-778. Doi https://doi.org/10.1038/nn.3983

Noble, K.G. y Giebler, M. A. (2020). The Neuroscience of Socioeconomic Inequality. Current Opinions in Behavioral Sciences, 36, 23-28. doi: 10.1016/j.cobeha.2020.05.007

Organizacion de las Naciones Unidas (ONU) (2020). Informe de políticas: La educación durante la COVID-19 y después de ella. Organización de las Naciones Unidas. Recuperado de https://www.un.org/sites/un2.un.org/files/policy_brief__education_during_covid-19_and_beyond_spanish.pdf

Organización para la Cooperación y el Desarrollo Económicos (OCDE). (2020). Perspectivas Económicas de América Latina 2020: transformación digital para una mejor reconstrucción. Recuperado de https://www.cepal.org/es/publicaciones/46029perspectivas-economicas-america-latina-2020-transformacion-digital-mejor 
Organización para la Cooperación y el Desarrollo Económicos (OCDE). (2021). OECD Skills Outlook 2021. Learning for Life. Recuperado de https://doi.org/10.1787/0ae365b4-en

Pierse, Nevil., Carter, Kristie., Bierre, Sarah., Law, David., and Howden-Chapman, Philippa. (2016). Examining the role of tenure, household crowding and housing affordability on psychological distress, using longitudinal data. Journal of Epidemiology and Community Health, 70(10), 961-966. https://doi.org/10.1136/jech-2015-206716

Pinker, Steven. (2018). Enlightenment now: The case for reason, science, humanism, and progress. Viking, an imprint of Penguin Random House LLC.

Programa Estado de la Nación (PEN). (2019). Séptimo Informe Estado de la Educación. Programa Estado de la Nación en Desarrollo Humano Sostenible.

Programa Estado de la Nación (PEN). (2020). ¿Cómo ha sido el contacto entre docentes y estudiantes en la nueva modalidad de clases a distancia? Recuperado de https://cutt.ly/9WxIJdT

Raver, C. C., Blair, C., Willoughby, M., and Family Life Project Key Investigators. (2013). Poverty as a predictor of 4-year-olds' executive function: New perspectives on models of differential susceptibility. Developmental Psychology, 49(2), 292-304. Doi https://doi.org/10.1037/a0028343

Romeo, Rachel R., Segaran, Joshua., Leonard, Julia A., Robinson, Sydeny T., West, Martin R., Mackey, Allyson P., Yendiki, Anastasia., Rowe, Meredith. L., and Gabrieli, John D. E. (2018). Language Exposure Relates to Structural Neural Connectivity in Childhood. The Journal of Neuroscience, 38(36), 7870-7877. https://doi.org/10.1523/JNEUROSCI.048418.2018

Stark, Oded., Micevska, Maja., and Mycielski, Jerzy. (2009). Relative poverty as a determinant of migration: Evidence from Poland. Economics Letters, 103(3), 119-122. Doi https://doi.org/10.1016/j.econlet.2009.02.006

Steele, C. M., and Aronson, J. (1995). Stereotype threat and the intellectual test performance of African Americans. Journal of Personality and Social Psychology, 69(5), 797-811.

Tawakol, Ahmed., Osborne, Michael T., Wang, Ying., Hammed, B., Tung, B., Patrich, T., Oberfeld, Blake., Ishai, Amorina., Shin, Lisa M., Nahrendorf, Matthias., Warner, Erica T., Wasfy, Jason., Fayad, Zahi A., Koenen, Karestan., Ridker, Paul M., Pitman, Roger K., and Armstrong, Katrina A. (2019). Stress-Associated Neurobiological Pathway Linking Socioeconomic Disparities to Cardiovascular Disease. Journal of the American College of Cardiology, 73(25), 3243-3255. Doi https://doi.org/10.1016/j.jacc.2019.04.042

Troller-Renfree, Sonya V., Brito, Natalie H., Desai, Pooja M., Leon-Santos, Ana G., Wiltshire, Cynthia A., Motton, Summer N., Meyer, Jerrold S., Isler, Joseph., Fifer, William P., and Noble, Kimberly G. (2020). Infants of mothers with higher physiological stress show alterations in brain function. Developmental Science, 23(6), e12976. Doi https://doi.org/10.1111/desc.12976

UNESCO. (2020). Inclusion and education, all means all. https://unesdoc.unesco.org/ark:/48223/pf0000373718 
UNICEF (2020). COVID-19 Education Response: Reaching the Most Vulnerable Children. UNICEF. Recuperado de https://www.unicef.org/lac/en/media/14531/file

Revista indizada en
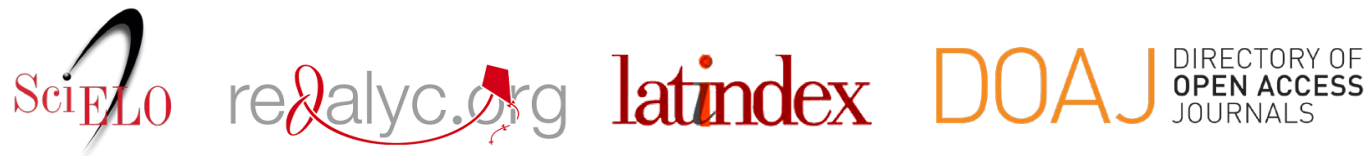

Distribuida en las bases de datos:
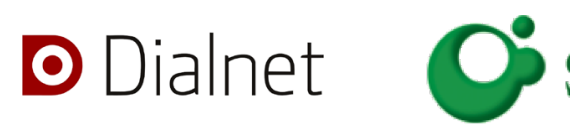
SHERPA/RøMEO

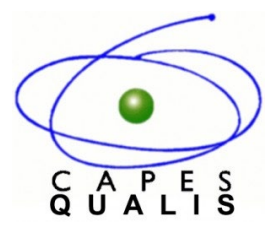

MIAR 